\title{
How are Cosmic Photons Redshifted?
}

\author{
H.-J. Fahr ${ }^{1 *}$ and M. Heyl ${ }^{2}$ \\ ${ }^{1}$ Argelander Institut für Astronomie, Universität Bonn \\ Auf dem Hügel 71, 53121 Bonn, Germany \\ ${ }^{2}$ Deutsches Zentrum für Luft und Raumfahrt (DLR) \\ Königswinterer Str. 522 - 524, 53227 Bonn, Germany \\ Email: hfahr@astro.uni-bonn.de
}

\begin{abstract}
According to present cosmological views, the energy density of CMB (Cosmic Microwave Background) photons, freely propagating through the expanding cosmos, varies proportional to $1 / S^{4}$ with $S$ being the scale factor of the universe. This behavior is expected because General Theory of Relativity, in application to FLRW- (Friedmann-Lemaitre-Robertson-Walker) cosmological universes, leads to the conclusion that the photon wavelengths increase during their free passage through the spacetime metrics of the universe by the same factor as does the scale factor $S$. This appears to be a reasonable explanation for the presently observed Planckian CMB spectrum with its actual temperature of about $2.7 \mathrm{~K}$, while at the time of its origin after the last scattering during the recombination phase its temperature should have been about $3000 \mathrm{~K}$, at an epoch of about $380 \mathrm{ky}$ after the Big Bang, when the scale of the universe $S_{\mathrm{r}}$ was smaller by roughly a factor of $S / S_{\mathrm{r}}=1+z_{\mathrm{r}}=1100$ compared to the present scale $S=S_{0}$ of the universe. In this paper we start from putting the question whether the scale-behavior of the CMB energy density that enters the energy-momentum tensor of the field equations describing the expanding universe is really falling off like $S^{-4}$ and, if in fact a deviation from a behavior according to $S^{-4}$ would occur, why do we nevertheless presently observe a CMB energy density which appears to be in accordance with such a $S^{-4}$-scaling? This question arises from another basic and fundamental question, namely: Can we really assume that the wavelength of the freely propagating photon during its travel all the way along its light geodetic is permanently affected by the expansion of the universe, i.e continuously recognizes the expansion of the cosmic scale $S$ ? With other words: Do freely propagating photons really undergo a permanent change of their wavelengths when freely traveling through cosmic space-time or is the observationally apparent energy loss of cosmologically red-shifted photons an effect which only occurs just in the moment of photon registration at some specific world point? If the latter would prove to be true, then it would mean that the energy density of freely propagating, non-interacting CMB photons, due to non-changing, conserved wavelengths, is behaving with respect to cosmic scale variation different from conventional expectations, but rather would turn out to behave just like the energy density of matter, namely according to $S^{-3}$. Hence the photon part of the energy momentum tensor would become different and associated solutions of FLRW-equations would undergo corresponding modifications. In consequence, the CMB energy density as far as it enters the energy-momentum tensor generated by freely propagating CMB photons during the expansion period of the universe after the recombination era would no longer become negligible for the cosmic dynamics, since its value would stay in the same order of magnitude as that of baryonic or dark matter.
\end{abstract}

Keywords: Cosmology, dark matter - cosmology, cosmic microwave background

\section{Introduction}

In the following considerations we start from the basis of both Einstein's Special and General Theory of Relativity (STR and GTR) where it is stated that for a photon moving with the velocity of light $c$ the so-called proper time increment (time increment $\mathrm{d} \tau_{0}$ in the photon proper system) vanishes, i.e. $\mathrm{d} \tau_{0}=0$ (see e.g. [1]; [2]; [3]; [4]). If this statement needs to be taken serious, then one seriously has to discuss the consequences of it and the conclusions to be drawn there from. Obviously it would mean that a photon moving along a geodetic trajectory (i.e. along its light geodetic) from spacepoint A to spacepoint B in its own reference system does not require proper time to cover this passage. Of course this should be 
true only in its individual reference system. In other words: the photon starts at some worldtime $t_{\mathrm{A}}$ in a spacepoint $\mathrm{A}$ and arrives later at a spacepoint $\mathrm{B}$ at a worldtime $t_{\mathrm{B}}$, however, this all happens while in the individual proper frame of this photon no time has lapsed, i.e. the light geodetic is an isochronal line for the individual photon.

The question then arises: How can a photon, moving freely in a cosmological spacetime, react to the change of the dynamical spacetime geometry, if in its proper system no time lapses? Should not its physical state be strictly preserved in the case of no lapse of time? We presume that due to the lack of proper time lapse the photon will simply not have the chance to react to dynamical spacetime geometry in the generally expected form, because without lapse of time nothing, i.e. no change can happen in the photons' reference frame. The consequences of that presumption, if confirmed as true, are enormous, meaning for instance that a photon freely propagating in cosmological spacetime, i.e. in a free falling system does not change its proper state, i.e. its energy and spin and, when seen in the wave picture of the photon, also its wavelength. In the photon frame no change of the photon state should happen, neither due to a change of an environmental gravitational potential, nor due to the expansion of the universe, i.e. any change of the cosmological metrics. The photon at free propagation simply would preserve its physical state which it got at the moment of its generation from an electromagnetic event, like emission from a stellar surface or from the CMB horizon. The so-called and generally believed cosmological redshift of its wavelength in an expanding universe ([3]; [5]; [6]; [7]; [8]) would thus simply have to be caused by the cosmic observer that measures the photon frequency with its local world clock (i.e. spectrometer), comparable perhaps with the special relativistic Doppler shift which results from the observer's relative motion with respect to the photon frame, i.e. the effect of the observer.

In the following sections of this paper we would like to explain more in detail the physical arguments behind the above idea. We then calculate the effects of the proposed constant wavelength of freely propagating photons (we call them "free photons" in the rest of the paper) in the cosmic era after recombination $\left(z \leq z_{\mathrm{r}} \approx 1100\right)$. Finally we discuss the implications that are connected with nonredshifted photons entering the energy momentum tensor $T_{\mathrm{ik}}$ of Robertson-Walker universes and resulting cosmological expansion solutions.

\section{Theory of the Cosmological Redshift}

In a universe with Robertson-Walker metric the world line element $\mathrm{d} l$ is given by (see [4])

$$
\mathrm{d} l^{2}=c^{2} \mathrm{~d} t^{2}-S^{2}(t)\left[\frac{\mathrm{d} r}{1-k r^{2}}-r^{2} \mathrm{~d} \Omega^{2}\right] .
$$

As is well known from SRT, but valid also in GRT, for the wordline of a photon, a so-called lightgeodetic, one has

$$
\mathrm{d} l^{2}=c^{2} \mathrm{~d} \tau_{0}^{2}=0
$$

meaning that in the proper system of the photon the proper time increment $\mathrm{d} \tau_{0}$ vanishes, implying that while the photon is propagating through space in its reference system no proper time is running, i.e. the photon in its proper system does not age, i.e. it consequently has to conserve its properties. That obviously must mean that during the passage of such a photon through cosmic spacetime this photon cannot and does not change its identity, though this photon on its light geodetic reaches worldpoints of different world ages. This implies that the photon does not change its physical signatures or characteristics like e.g. its energy or its angular momentum, i.e. during its free flight along light geodetics it conserves those properties with which it was generated. This is because an object, as also a photon in the quantum duality view seen as a particle, cannot change its physical signatures under any physical action which does not have a finite increment in time $\mathrm{d} \tau_{0}$ to act.

To give more credit to this view we would like to mention the following aspect: The above argumentation is well familiar to SRT scientists, is standardly used to carry out time synchronization at different places and can easily be compared and controlled with the standard practice of synchronizing times in a Schwarzschildian spacetime geometry as shown in subsection 2.2 . 


\subsection{As a Reminder: Redshift in a Schwarzschildian Metric}

We shall follow here the presentation given by [1] and shall first pick up the most important idea presented there. Assuming that the gravitational field of the central Schwarzschildian mass acts on a photon freely propagating in the predetermined spacetime geometry then allows to describe the photon propagation as happening in a free falling, inertial system (photon in a free falling cabin). As [1] clearly and correctly states, in that free falling cabin (inertial system = ff-system) no change in frequency of the propagating photon occurs, i.e. the free falling photon does not change its frequency in this system. The question only is how this fact can be transfered into the non-inertial system, i.e. into the fixed Scharzschildian coordinate system. The argumentation then is that the free-falling system (cabin) has first to be replaced by an equivalent inertial system calculating the velocity $\mathrm{d} u$ that the free fall over a propagation distance $\mathrm{d} l$ induces due to the acting gravitational acceleration $g=\mathrm{d} \Phi / \mathrm{d} r$. Neglecting second order corrections one finds that the equivalent velocity increase is given by

$$
\mathrm{d} u=(\mathrm{d} l / c) g=(\mathrm{d} l / c)(\mathrm{d} \Phi / \mathrm{d} r) .
$$

Transforming now from the equivalent inertial system (ff-system) into the fixed Schwarzschildian system (ss-system) needs the SRT frequency transformation from the system moving with $\mathrm{d} u$ into the system at rest,i.e. leading to

$$
\begin{aligned}
\nu_{\mathrm{ss}}= & \nu \sqrt{(1+\mathrm{d} u / c) /(1-\mathrm{d} u / c)} \simeq \\
& \nu \sqrt{(1+\mathrm{d} u / c)^{2}}=\nu(1+\mathrm{d} u / c)
\end{aligned}
$$

or

resulting in

$$
\nu_{\mathrm{ss}}-\nu=\nu(\mathrm{d} u / c)
$$

$$
d \nu / \nu=\left(\nu_{\mathrm{ss}}-\nu\right) / \nu=(\mathrm{d} u / c)
$$

leading with $d \nu / \nu=d \ln \nu$ and $\mathrm{d} l \cdot g / c=-\mathrm{d} r \cdot \operatorname{grad} \Phi / c=\mathrm{d} \Phi$ to the well known result that

$$
\begin{array}{r}
\nu_{s s}=\nu \cdot \exp \left[\int\left(\mathrm{d} l / c^{2}\right)(\mathrm{d} \Phi / \mathrm{d} r)=\right. \\
\nu \cdot \exp \left[-\frac{1}{c^{2}} \int_{\Phi_{1}}^{\Phi_{2}} \mathrm{~d} \Phi\right]=\nu \cdot \exp \left[-\frac{\Delta \Phi_{1,2}}{c^{2}}\right] .
\end{array}
$$

That finally again expresses, also in the case of this Scharzschildian spacetime metric, the remarkable fact that a photon, - not while freely propagating over a region connected by a potential difference $\Delta \Phi_{1,2}$ -, but only, when measured in the static Schwarzschild frame, reflects a frequency shift of the above mentioned order.

This context is commonly interpreted as saying: having two clocks at different positions $r_{1}$ and $r_{2}$ within a Schwarzschild metric, one must expect a difference in the beats of the time $t_{1}$ and $t_{2}$ at these different positions. Since photons on spacepoint connecting geodetics are perfect time-synchronizers in spacetime, they conserve the time digits. If thus a photon transports time signatures connected with its frequency $\nu_{1}$ at $r_{1}$ to the spacepoint $r_{2}$, then these time signatures arriving at $r_{2}$ are registered there by the local beat of the time, i.e. by

$$
\nu_{2}=\nu_{1} \exp \left[-\frac{\Delta \Phi_{1,2}}{c^{2}}\right] .
$$

This phenomenon does not mean that the photon changes its frequency while passing along its light geodetic, rather the photon is emitted with its genuine frequency $\nu_{1}$ and keeps it in the ff-system, but when registered at a place $r_{2}$ its frequency appears, as if it had locally changed to the frequency $\nu_{2}$. This means the so-called Mößbauer effect does not prove the change of the photon frequency in free geodetic flights, but proves that the new evaluation of the original frequency $\nu_{1}$ with a new local clock detuned with respect to the one by which the photon originally was emitted. 


\subsection{As an Analogy: Redshift Induced by a Cosmic Potential}

As one can easily show (see e.g. [9]) in a homogeneous universe with vanishing curvature (i.e. $k=0$ !) one can formally introduce a cosmic gravitational potential which can be derived from the first of the two Friedman equations and takes the simple form:

$$
\Phi_{\cos }(R)=\frac{8 \pi G}{3} \rho R^{2} .
$$

Here $G$ is the gravitational constant, $\rho$ denotes the cosmic matter density if dark matter and dark energy are neglected, and $R=R(t)$ is the actual scale of the universe at world time $t$. Assuming that no dark energy has to be taken into account $(\Lambda=0$ !), and that only conservatively behaving representations of matter (i.e. particles with conserved total numbers; no decay or creation of matter!) have to be taken into account, then matter density scales inversely proportional with cosmic volume, hence with $R^{-3}$, and the above potential consequently can be written in the form

$$
\Phi_{\cos }(R)=\frac{8 \pi G}{3} \frac{M}{R}
$$

where $M$ has been introduced as the total and constant mass of the universe. The above expression then shows that, the larger the universe grows, the smaller its cosmic potential, finally approaching $\Phi_{\cos }(R \rightarrow \infty)=0$.

This situation can be compared with that of clocks at different positions in a stationary central gravitational field (see section 2.1). As is well known the beat of the clocks at different positions of a gravitational potential in this case is detuned (see Rindler, 1977). Analogously based, however, on the above cosmic potential, the cosmic beat of the time, in an inertial system should be subject to the following law

$$
t=t_{0} \exp \left[-\frac{\Phi(R)}{c^{2}}\right] .
$$

From that one derives the following relation between frequencies of CMB photons $\nu_{\text {rek }}$ near the recombination period, when the scale of the universe was $R=R_{r e k}$, and the associated frequencies $\nu$ of such CMB photons registered at the present time:

$$
\frac{\nu-\nu_{r e k}}{\nu}=\frac{\Delta \Phi_{e f f}}{c^{2}}=-\frac{8 \pi G}{c^{2}}\left[\rho_{r e k} R_{r e k}^{2}-\rho R^{2}\right]=-\frac{8 \pi G M}{c^{2}}\left[\frac{1}{R_{r e k}}-\frac{1}{R}\right] .
$$

Here the potential energy $m_{\nu} \Delta \Phi_{\text {eff }}$ has been considered to be small with respect to the photon energy itself $m_{\nu} c^{2}$. Neglecting the small terms in the brackets on LHS and RHS one thus can find:

$$
\frac{\nu_{r e k}}{\nu}=\frac{8 \pi G M}{c^{2} R_{r e k}} .
$$

According to the above relation, the unchanged frequency of the CMB photon from the recombination phase would then, when registered today by a local clock (i.e. spectrometer), appear with a frequency $\nu$ given by

$$
\nu=\nu_{r e k} \frac{c^{2} R_{r e k}}{8 \pi G M}
$$

\subsection{Alternative View: Redshift as Accumulated Doppler-Shift}

An alternative way to explain the expansion related redshift is to regard it as an accumulation of infinitesimal Doppler-Shifts along the photon's trajectory ([10]; [11]). The basic idea here is that the fractional frequency shift $\delta \nu / \nu$ for a photon which travels a distance $d=c \delta t$ within a time increment $\delta t$ is given by

$$
\delta \nu / \nu=\delta v / c=-(H d) / c=-H \delta t
$$


with $H$ the Hubble parameter and $v$ the recession velocity of the photon emitting galaxy $(v<<c)$. Now, using $H=\dot{S} / S$ with $S$ the scale parameter, we finally get

$$
\delta \nu / \nu=-\delta S / S
$$

the integration of which again leads to the well-known cosmological redshift equation

$$
\lambda=\lambda_{0} S(t) / S\left(t_{0}\right) .
$$

It should be pointed out that characteristic of the Doppler-Shift is a non-changing frequency/wavelength during the photon's travel from the emitter to the observer, no matter whether the relative velocity between emitter and observer is constant or not. This means that also the cosmological redshift - here seen as an accumulated Doppler-Shift - is just an effect which occurs in the moment of observation. During the travel of the photon its frequency/wavelength remains unchanged.

\subsection{Change of the Beat of Global Worldtime}

Hence we want to ask now, why the well-founded and well-believed phenomenon of a cosmological redshifting of a freely propagating photon can occur at all, if on the other hand the photon cannot change its physical state due to the lack of any time lapse in its ff-system. For that we go back to the line element of the freely propagating photon in a given dynamic cosmic spacetime geometry within the frame of the Friedmann-Lemaître-Robertson-Walker cosmology. With $\mathrm{d} \tau_{0}=\mathrm{d} \Omega=0$ we find from Eq. (1):

$$
\xi(r)=\int_{r_{0}}^{r} \frac{\mathrm{d} r}{\sqrt{1-k r^{2}}}=-c \int_{t_{0}}^{t} \frac{\mathrm{d} t}{S(t)} .
$$

Now it is generally assumed that a photon is an extended wave, allowing to write the analogue of the above equation not only for the beginning of the wavetrain (phase "a"), but also for the end of the wavetrain (phase "b"). Adopting then that during the time $\delta t_{0}=\lambda_{0} / c$ at the place of the photon emission the geometry of the universe has not changed, will then permit to write the following equation for the propagation of phase "b" of this photon

$$
\xi(r)=\int_{r_{0}}^{r} \frac{\mathrm{d} r}{\sqrt{1-k r^{2}}}=-c \int_{t_{0}+\delta t_{0}}^{t+\delta t} \frac{\mathrm{d} t}{S(t)}
$$

and one obtains as the difference of these two equations

$$
\frac{\delta t}{S(t)}=\frac{\delta t_{0}}{S\left(t_{0}\right)}
$$

or

$$
\frac{\delta t}{\delta t_{0}}=\frac{\lambda}{\lambda_{0}}=\frac{S(t)}{S\left(t_{0}\right)}
$$

meaning that the wavelength of the photon during its propagation has been changed according to the change in the universal scale $S(t)$ on its way. How to understand this?

If one says that the photon is characterized by a wavelength $\lambda_{0}$ which it keeps during all its free propagation, then it simply means the time increment $\delta t_{0}=\lambda_{0} / c$ is different from that $\delta t=\lambda / c$, because the time propagation rate (the beat of the time) has changed during the change in the world time between time $t_{0}$ and time $t$.

That can be interpreted as if the photon on its passage through cosmic spacetime keeps its identity, i.e. its wavelength $\lambda_{0}$, but when it passes over a detector at some spacepoint at worldtime $t$, then it needs a time

$$
\frac{\lambda}{c}=\frac{\lambda_{0}}{c} \frac{S(t)}{S\left(t_{0}\right)}
$$

Assuming a constant velocity of light this can be interpreted as saying that the beat of the global worldtime has changed between the events of emission and absorption of the photon.

All the findings in section 2 are the basis for the considerations presented in the next section. 


\section{Energy Density of Cosmic Photons after the Recombination Era}

According to the standard model of cosmology the spectral character of LTE- photons created during the phase of recombination just before the last photon scattering, say at $z_{\mathrm{r}} \approx 1100$ under conventional CMB assumptions, can be described by a Planck distribution with a temperature $T_{\mathrm{r}} \approx 3000 \mathrm{~K}$. Due to the expansion of the universe the wavelengths of the free CMB photons are usually assumed to increase and only because of that the spectrum stays Planckian, since then the Planck temperature of the CMB photons decreases according to $T(z)=(1+z) \cdot T_{0}$ (see e.g. [12]; [13]). This cooling is expected to explain the present-day CMB temperature $T(z=0)=T_{0} \approx 2.7 \mathrm{~K}$. For a Planck spectrum the energy density $\epsilon_{\gamma}$ of the CMB photons and the associated photon number density $n_{\gamma}$ are given by the well known equations (see [14]; [15]):

$$
\epsilon_{\gamma}=\frac{4 \sigma_{\mathrm{SB}}}{c} T^{4} \approx 0,00472 \mathrm{eV} \frac{T^{4}}{\mathrm{~cm}^{3}}
$$

with $\sigma_{\mathrm{SB}}$ denoting the Stefan-Boltzmann constant and $T$ given in $K$, and

$$
n_{\gamma}=\int_{0}^{\infty} \frac{\varepsilon_{\gamma}(\lambda) \lambda d \lambda}{h c} \approx 20,28 \frac{T^{3}}{\mathrm{~cm}^{3}}
$$

with $h$ the Planck constant and again $T$ given in $K$. In this cosmological "standard view" we can then for a present-day CMB temperature of $T_{0}=2.7 \mathrm{~K}$ expect an energy density $\epsilon_{\gamma, 0}$ of roughly

$$
\epsilon_{\gamma, 0} \approx 0,00472 \mathrm{eV} \frac{T_{0}^{4}}{\mathrm{~cm}^{3}} \approx 0.26 \mathrm{eV} / \mathrm{cm}^{3}
$$

and a photon number density $n_{\gamma, 0}$

$$
n_{\gamma, 0} \approx 20,28 \frac{T_{0}^{3}}{\mathrm{~cm}^{3}} \approx 400 / \mathrm{cm}^{3} .
$$

We now want to apply our new idea of a constant wavelength of each CMB photon at world times after the recombination era. Since the energy of single CMB photons does not change under the above assumption of a constant wavelength, the number density of the CMB photons changes as function of the inverse volume increase due to the expansion, i.e the ratio $\left(S_{r} / S_{0}\right)^{3}$ with $S_{r}=S\left(t_{r}\right)$ being the scale parameter at worldtime $t_{r}$ at the end of the recombination era and $S_{0}$ its reference value at the worldtime $t_{0}$, i.e. today. Thus, the present photon number density would then again be as in Eq. (24):

$$
n_{\gamma, 0}^{\lambda=\text { const. }}=20,28 \frac{T_{\mathrm{r}}^{3}}{\mathrm{~cm}^{3}}\left(\frac{S_{\mathrm{r}}}{S_{0}}\right)^{3} \approx 400 / \mathrm{cm}^{3} .
$$

Here $T_{r}$ is the temperature at the end of the recombination era $\left(T_{\mathrm{r}}=\left(1+z_{\mathrm{r}}\right) T_{0}\right)$. The present photon number density would be in fact the same, since it is now the increase of the scale parameter by a factor $S_{0} / S_{\mathrm{r}}=\left(1+z_{\mathrm{r}}\right) \approx 1100$ that reduces the number density to the same value that further above we had derived for the case of a conventionally decreasing CMB radiation temperature.

In contrast to that, under the new auspices of a constant wavelength the present-day cosmological photon energy density would, however, not be identical with the value given in Eq. (25), but for energyconserving CMB photon propagation now would be higher by a factor $\left(1+z_{\mathrm{r}}\right)$ because the photons have not lost energy during the expansion. Their energy density thus is the one valid at the recombination era, reduced by the reciprocal of the volume factor. With the assumption of a constant wavelength the present photon energy density at $S=S_{0}$ can therefore be written as:

$$
\epsilon_{\gamma, 0}^{\lambda=\text { const. }}=\frac{4 \sigma_{\mathrm{SB}}}{c} T_{\mathrm{r}}^{4}\left(\frac{S_{\mathrm{r}}}{S_{0}}\right)^{3} .
$$

Instead of Eq. (25) we then finally obtain:

$$
\begin{array}{r}
\epsilon_{\gamma, 0}^{\lambda=\text { const. }} \approx\left(1+z_{\mathrm{r}}\right) 0,26 \mathrm{eV} / \mathrm{cm}^{3} \approx \\
286 \mathrm{eV} / \mathrm{cm}^{3} \approx 286 \mathrm{MeV} / \mathrm{m}^{3} .
\end{array}
$$


At this point of the paper we like to strictly point out and strongly emphasize the following 2 points:

1. The above considerations, namely the scale behavior $\propto S^{-3}$ and the related results in the Eqs. (28) and (29), are valid only for freely propagating CMB photons. Then - instead of $S^{-4}$ - the $S^{-3}$-scaling of the photon energy density must be used for the free CMB photons to calculate the cosmic dynamics in the frame of the Friedmann equations (FLRW cosmology).

2. As soon as these CMB photons interact, e.g. with an observer, the detected wavelength will reflect the cosmological wavelength shift and show the well-known $S^{-4}$-scaling of the photon energy density due to the reasons explained in Section 2. Thus, an observer at the present time will always see a Planckian CMB spectrum with a temperature of $\approx 2.7 \mathrm{~K}$ !

Now it is extremely interesting to recognize that in comparison to the energy density given in Eq. (29) the energy density of visible, baryonic matter in the universe is about $\rho_{0, \mathrm{~B}} \approx 0,3 \mathrm{~m}_{\text {proton }} c^{2} / \mathrm{m}^{3} \approx$ $282 \mathrm{MeV} / \mathrm{m}^{3}$. Interestingly enough, both energy density values are nearly identical. In other words: the energy density of free CMB photons shows the same drop-off with scale as the energy density of the baryonic matter even in the present universe. One hence can conclude on the basis of assumptions made in this paper, that the energy density of free CMB photons at present is not negligible compared to the matter density but comparable in numbers, and this is valid not only for the cosmic epoch now, but should stay valid for the cosmological evolution into the past.

\section{Discussion and Conclusion}

We have based our investigations on the idea of a constant wavelength (energy) of freely propagating photons, i.e. photons that, while propagating through the universe, interact neither with each other nor with material particles like protons or electrons. The concept of zero-lapse of proper time for photons in both, STR and GTR, must lead in consequence to unaffected physical characteristics of photons propagating along light geodetics. This means that the wavelength does not continuously change during the free flight but remains constant. During this phase of free propagation the photon energy density simply scales according to $S^{-3}$ (i.e. a pure volume effect) which is then the relevant dependence to be taken serious for the input into the energy-momentum source tensor $T_{\mathrm{ik}}$ entering the calculation of the cosmic scale dynamics with Robertson-Walker-Friedmann-Lemaître equations. In other words: the energy density of freely propagating cosmic photons (no matter if CMB or galactic photons) is changing like the photon number density of these photons according to $S^{-3}$, since the energy of each individual photon does not change with the expansion. What happens is, that the clock of the observer is cosmologically detuned with respect to that of the emitter, i.e. photons communicating between emitter and observer are differently qualified at their origin and at their absorber (observer). As soon as an observer interacts with the photons, i.e. analyzes the wavelength or measures the frequency of them, they appear, as if their energy density had followed the conventionally believed $S^{-4}$ law, because these interactions occur at another worldtime compared to that of their origin. In consequence one must realize that the observed photon energy density $\left(\propto S^{-4}\right)$ differs from the energy density of free photons, the latter of which, however, is relevant for the cosmic dynamics $\left(\propto S^{-3}\right)$. For the universe of present days the energy of cosmic photons cannot be neglected with respect to that of baryons, and this situation should be continuing to hold in the cosmological times to come.

\section{References}

1. M. Rindler, Essential Relativity. Springer, New York, 1977.

2. R. C. Tolman, Relativity, Thermodynamics and Comology. Dover, Toronto, 1987.

3. C. W. Misner, K. S. Thorne, and J. A. Wheeler, Gravitation. Freeman, San Francisco, 1973.

4. H. F. M. Goenner, Einführung in die Spezielle und Allgemeine Relativitätstheorie. Spektrum Akademischer Verlag, Heidelberg, 1996.

5. M. Berry, Kosmologie und Gravitation. Teubner, Stuttgart, 1990.

6. R. Sexl and H. Urbantke, Relativität, Gruppen, Teilchen. Springer, Wien, 1987.

7. J. A. Peacock, Cosmological Physics. Cambridge/MA: Cambridge University Press, 1999. 
8. J. C. Pecker and J. P. Vigier, Observational Cosmology. IAU, Bejing, 1987, vol. 507.

9. H. J. Fahr and M. Heyl, "About universes with scale-related total masses and their abolition of presently outstanding cosmological problems," $A N$, vol. 328, no. 2, 2007.

10. E. F. Bunn and D. W. Hogg, "The kinematic origin of the cosmological redshift," arXiv: 0808.1081v2, 2008.

11. J. A. Peacock, "A diatribe on expanding space," arXiv: 0809.4573v1, 2008.

12. H. J. Fahr and J. Zoennchen, "The writing on the cosmic wall is there a straightforward explanation of the cosmic microwave background?" Ann. Phys., vol. 18, no. 10-11, p. 699, 2009.

13. H. J. Fahr and M. Sokaliwska, "Remaining problems in the interpretation of the cosmic microwave background," Physics Research International (Hindawi), vol. 1, no. Article 503106, 2015.

14. D. W. Sciama, Modern Cosmology. Cambridge University Press, New York, 1971.

15. P. J. E. Peebles, Principles of Physical Cosmology. Princeton University Press, Princeton, 1993. 